УДК 629.4.027.11:338.27

\title{
РОЗРОБКА МОДЕЛІ ПРОГНОЗУВАННЯ ВІДЧЕПЛЕНЬ ВАГОНІВ ПО НЕСПРАВНОСТЯХ БУКСОВИХ ВУЗЛІВ
}

\author{
К-т техн.наук В.М. Петухов, магістрант А.В.Депутат
РАЗРАБОТКА МОДЕЛИ ПРОГНОЗИРОВАНИЯ ОТЦЕПОК ВАГОНОВ ПО НЕИСПРАВНОСТЯМ БУКСОВЫХ УЗЛОВ

\section{К-т техн.наук В.М. Петухов, магистрант А.В.Депутат
DEVELOPMENT MODEL OF PROGNOSTICATION OF THE UNCOUPLING OF CARRIAGES ON DISREPAIRS BUKSOVYKH KNOTS

\section{Cand. of techn. sciences V.M. Petukhov, master student A.V. Deputat}

Одним з безлічі важливих вузлів $і$ деталей конструкиії вагону є ходові частини рухомого складу, а саме буксові вузли. У цій роботі розглянутий процес прогнозування, який припускає виявлення можливих альтернатив розвитку в перспективі для обгрунтованого їх вибору і ухвалення оптимального рімення.

Ключові слова: вагон, буксовий вузол, прогнозування.

Одним из множества важных узлов и деталей конструкиии вагона являются ходовые части подвижного состава, а именно буксовые узльл. В данной работе рассмотрен процесс прогнозирования, который предполагает выявление возможных альтернатив развития $в$ перспективе для обоснованного их выбора и принятия оптимального решения.

Ключевые слова: вагон, буксовый узел, прогнозирование.

Solution of important research and practice task consisting of development of model of prognostication of uncoupling of carriages on the disrepairs of buksovye knots for determination of necessity of enterprises of carriage economy in awaiting-parts is in-process offered. The offered model will allow the processes of determination of supplies of wheelpairs, promote efficiency of the existent systems of logistical support of enterprises.

For the practical calculations of prognosis of uncoupling on the disrepairs of buksovye knots there is the approach based on the use of presentation of model equalization with a minimum error.

The worked out methodology allows to forecast the number of refuses of buksovye knots and on this prognosis to optimize a supply, for example, of wheelpairs. Further researches must be sent to development of methodologies of prognostication of requirement in awaiting-parts for the repair enterprises of carriage economy, storages and perfection of management methods by supplies these.

Keywords: carriage, buksovye knot, prognostication.

Введение. Отказы грузовых вагонов и связанные с этим задержки в движении поездов являются одним из основных факторов, дестабилизирующих эксплуатационную работу железнодорожного транспорта[1,7,8,9]. Для оперативной замены неисправных узлов на пунктах технического обслуживания вагонов имеется неснижаемый запас запасных частей. В масштабах даже одной дороги это довольно значительное количество(рис.1).

В современных условиях реформирования железнодорожной отрасли большое значение имеет оптимизация количества запасных частей, а также прогнозирование их потребности для планирования работы ремонтных 
предприятий и финансирования закупок новых изделий.

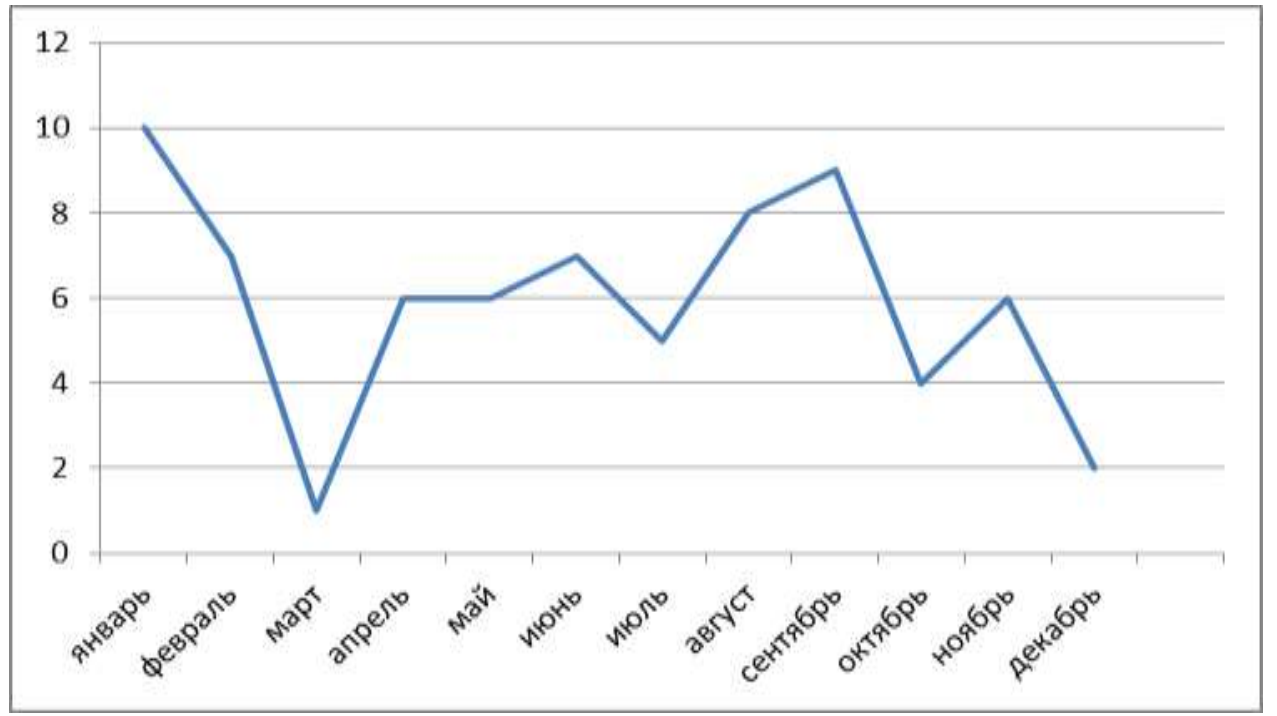

Рисунок 1- График количества отцепок по неисправностям буксовых узлов на Южной железной дороге

Постановка проблемы в общем виде и ее связь с важнными научными и практическими задачами

Таким образом, для решения проблемы оптимизации запасных частей для отцепочного ремонта вагонов требуется научный подход для создания прогнозной модели, которая бы позволила бы решать практические задачи по определению оптимального количества запасных частей.

Определение цели и задачи исследования

Целью работы является разработка модели прогнозирования отказов букс для совершенствования планирования запасных частей грузовых вагонов.
Проведение

исследований направленных на достижение цели потребовало решения следующих задач.

1. Обработка статистических данных об отказах буксовых узлов на ЮЖД.

2. Выбор и обоснование прогнозной модели.

Анализ последних исследований и публикаций

Анализ существующих методов планирования фонда запасных частей на предприятиях показал, что при обеспечении предприятий запасными частями могут использоваться методы, представленные на рис. 2.

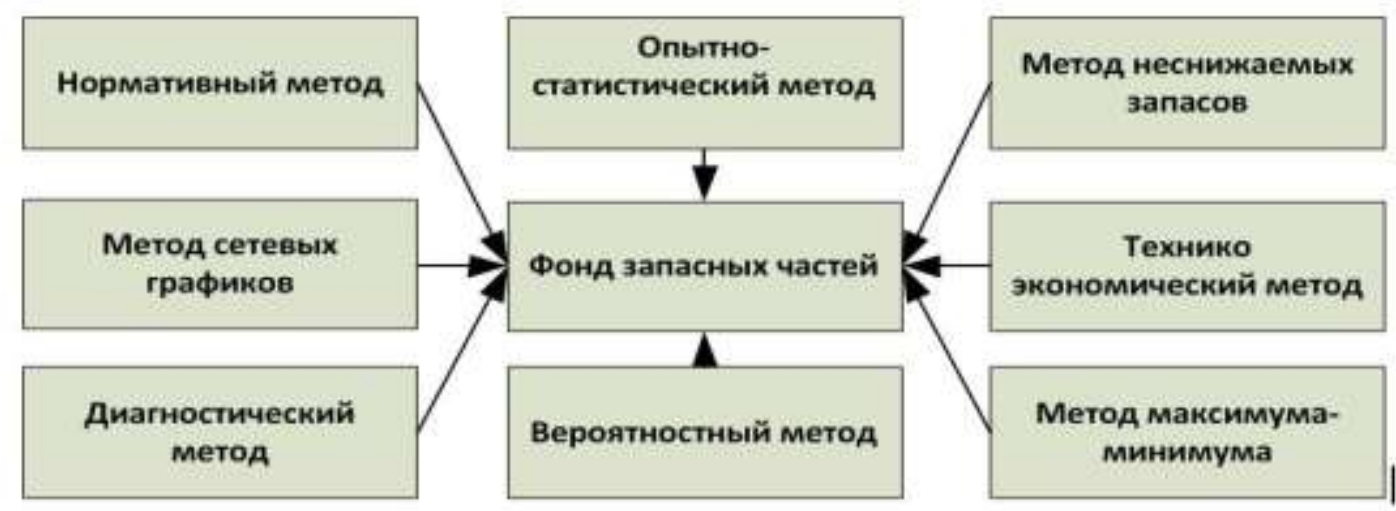

Рисунок - 2 Методы планирования фонда запасных частей.

Наиболее приемлемые методы для разработаны для крупных автотранспортных использования в вагонном хозяйстве предприятий[2,4,11,12]. Доступным и 
дающим адекватные результаты является метод на основе анализа временно́го ряда[3,5,6,10].

Основная часть исследования. Одна из важных проблем вагонного хозяйства определить количество запасных частей для отцепочного текущего ремонта в условиях неопределенности.

Инструментом минимизации неопределенности служит прогнозирование, а прогнозом называют научно обоснованный вывод о будущих событиях, о перспективе развития процессов, о возможных следствиях принятых решений.

Прогнозирование предусматривает систему научных сведений, использование методов и приемов с разной степенью формализации, согласованность отдельных выводов и оценок относительно будущего развития процесса.

В мировой практике прикладного прогнозирования используют разные методы: статистические (прогнозная экстраполяция), ф функциональноиерархические (прогнозные сценарии), методы структурной аналогии, имитационного моделирование, экспертные оценки. Каждый метод имеет свои особенности, положительные качества и недостатка, свои границы использования.

Другой

статистического

прогноза

особенностью горизонт прогноза называют периодом упреждения. Время периода упреждения зависит от специфики объекта прогнозирования, интенсивности динамики, продолжительности действия выявленных закономерностей и тенденций.

Прогнозный результат на период упреждения можно представить одним числом (точечный прогноз) или интервалом значений, к которому с определенною вероятностью принадлежит прогнозная величина (интервальный прогноз).

Статистические

прогнозы основываются на гипотезах о стабильности значений величины, которые прогнозируется; закона ее распределения; взаимосвязей с другими величинами и т.п.
Основной инструмент прогнозирования экстраполяция.

Суть прогнозной экстраполяции заключается в распространении закономерностей, связей и отношений, выявленных в $i$-му периоде, за его границы.

Это делает возможным осуществлять краткосрочное прогнозирование состояния объекта, используя метод на основе анализа временно́го ряда.

Такое прогнозирование предполагает не только качественное предсказание, но и достаточно точное количественное измерение вероятных возможностей ожидаемых значений признака. Для данной цели важно, чтобы прогностическая модель имела достаточную точность или допустимо малую ошибку прогноза.

Как и большинство других видов анализа, анализ временных рядов предполагает, что данные содержат систематическую составляющую (обычно включающую несколько компонент) и случайный шум (ошибку), который затрудняет обнаружение регулярных компонент. Большинство методов исследования временных рядов включает различные способы фильтрации шума, позволяющие увидеть регулярную составляющую более отчетливо (рис. 3 ).

Большинство регулярных составляющих временных рядов принадлежит к двум классам: они являются либо трендом, либо сезонной составляющей. Тренд представляет собой общую систематическую линейную или нелинейную компоненту, которая может изменяться во времени. Сезонная составляющая - это периодически повторяющаяся компонента. Оба эти вида регулярных компонент часто присутствуют в ряде одновременно.

Для выбора прогностической модели предлагается ряд типовых временных рядов [3] (рис.4).

Для решаемой задачи более всего подходит, это видно из рисунка 4, ряд типа С. Такой ряд достаточно точно описывается моделью

$$
(1-0,8 B)(1-B) z_{t+l}=a_{t+l},
$$




\begin{tabular}{l}
\hline \hline где $B-$ оператор сдвига назад, \\
определяемый как $B z_{t}=z_{t-1} ;$ \\
$z_{t}-\quad$ значение контролируемой \\
величины в момент времени $t$; \\
$z_{t-1}-\quad$ значение контролируемой \\
величины в предыдущий момент времени $t-1$ \\
$z_{t+l}-$ значение контролируемой \\
величины через время упреждения $l, l=$ \\
$1,2 \ldots n ;$
\end{tabular}

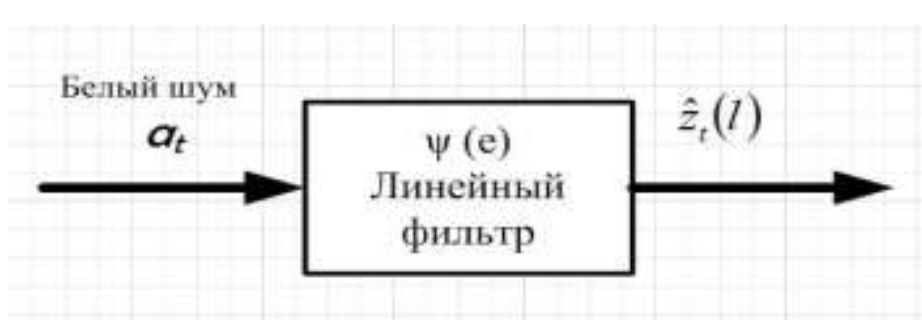

Рисунок 3 - Модель прогнозирования

$a_{t+l}-$ независимый импульс, «белый
шум». Эти импульсы - реализации случайных величин с фиксированным распределением, которое обычно предполагается нормальным с нулевым средним и дисперсией $\sigma_{a}^{2}$.
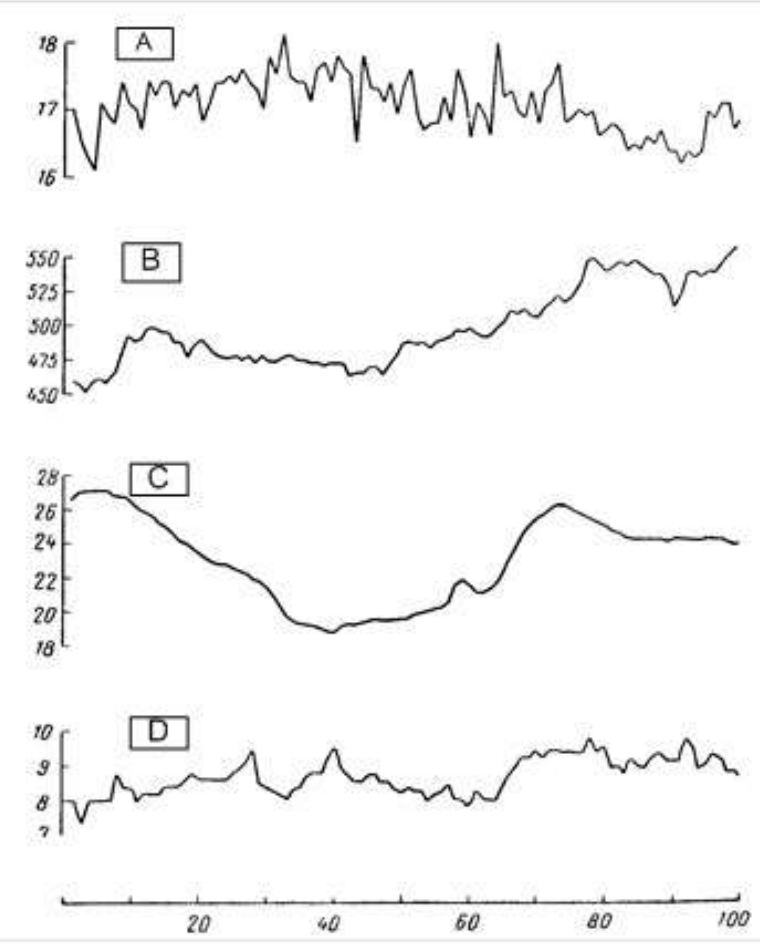

Рисунок 4 -Типичные временные ряды

Предполагается, что наблюдения производились в дискретные, примерно равноотстоящие друг от друга моменты времени. Температура шейки оси $z_{t}$, на в момент времени $t$ и температура $z_{t-1}, z_{t-2}, z_{t-3}$ и т.д. могут быть использованы для прогноза с упреждением $\boldsymbol{l}$. Функция $\hat{z}_{t}(l), l=1,2 \ldots$ дающая в момент $t$ прогнозы для всех будущих времен упреждения, называется прогнозирующей функцией в момент $t$. Цель - получить такую прогнозирующую функцию, у которой среднее значение квадрата отклонения $z_{t+l}-\hat{z}_{t}(l)$ истинного от прогнозируемого значения является наименьшим для каждого упреждения $l$.

В дополнение к вычислению наилучшего прогноза необходимо также указать его точность, чтобы можно было оценить риск, связанный с решениями, основанными на прогнозировании. Точность прогноза может быть выражена вероятностными пределами по обе стороны 
от каждого прогнозируемого значения. Эти пределы можно вычислить для любого набора вероятностей, например для 95\%. Смысл этих пределов в том, что значение временного ряда, которое появится в соответствующее время, с указанной вероятностью будет лежать внутри этих пределов.

\section{Прогнозы} среднеквадратичной

$$
\begin{aligned}
& \text { с минимальной } \\
& \text { ошибкой можно } \\
& \hat{z}_{t}(1)=1,8 z_{t}-0,8 z_{t-1}, \\
& \hat{z}_{t}(2)=1,8 \hat{z}_{t}(1)-0,8 z_{t}, \\
& \hat{z}_{t}(l)=1,8 \hat{z}_{t}(l-1)-0,8 \hat{z}_{t}(l-2), \quad l=3,4,5, \ldots
\end{aligned}
$$

Чтобы вычислить новые прогнозы путем коррекции старых, требуется определить веса $\psi_{1}, \psi_{2}, \ldots \psi_{l-1}$.

Для используемой модели веса вычисляются рекуррентным способом:

$$
\begin{aligned}
& \psi_{1}=1,8, \\
& \psi_{j}=1,8 \psi_{j-1}-0,8 \psi_{j-2}, j=2,3,4, \ldots
\end{aligned}
$$

Как только становится известно новое значение температуры буксы $z_{t+1}$, появляется возможность пропорционально подправить прогноз. Для этого определяется импульс $a t$, определяемый как разность $a_{t}=z_{t}-\hat{z}_{t-1}$

Тогда уточненный прогноз определяется следующим выражением:

$$
\hat{z}_{t+1}(l)=\hat{z}_{t}(l+1)+\psi_{l} a_{t}
$$

Из предположения, что $a$ подчиняются нормальному закону, следует, что при известных значениях процесса до момента $\boldsymbol{t}$ условное распределение вероятности $\quad p\left(z_{t+l} / z_{t}, z_{t-l}, \ldots\right)$ будущего значения процесса $z_{t+l}$ будет также нормальным со средним значением $\hat{z}_{t}(l)$ и стандартным отклонением $\left\{1+\sum_{j=1}^{l-1} \psi_{j}^{2}\right\}^{1 / 2} \sigma_{a}$ получить непосредственно из представления модели в виде разностного уравнения. Для практических вычислений прогнозов температуры буксы наиболее приемлемым является подход, основанный на использовании представления модели разностным уравнением[2]. Такие прогнозы в момент $t$ будут иметь вид:
Приближенные $\quad(1-\quad \varepsilon) \%$-ные вероятностные пределы $z_{t+l}(-)$ и $z_{t+l}(+)$ для $z_{t+l}$ будут иметь вид:

$$
z_{t+l}( \pm)=\hat{z}_{t}(l) \pm u_{\varepsilon / 2}\left\{1+\sum_{j=1}^{l-1} \psi_{j}^{2}\right\}^{1 / 2} s_{a}
$$

Пределы $z_{t+l}(-) \quad$ и $\quad z_{t+l}(+)$ интерпретируются следующим образом. Если известна информация о временном ряде к моменту $t$, то с вероятностью $1-\varepsilon$ наблюденное значение $z_{t+l}$ будет заключено в этих пределах, т. е,

$$
\operatorname{Pr}\left\{z_{t+l}(-)<z_{t+l}<z_{t+l}(+)\right\}=1-\varepsilon
$$

\section{Выводы из исследование и перспективы, дальнейшее развитие в данном направлении.}

Предложенная методика позволяет прогнозировать число отказов буксовых узлов и на этом прогнозе оптимизировать запас колесных пар. Дальнейшие исследования должны быть направлены на разработку методик прогнозирования потребности в запасных частях для ремонтных предприятий вагонного хозяйства, складов и совершенствование методов управления запасами этих систем.

\section{Список використаних джерел}

1. Аналіз стану безпеки руху на залізницях України у 2013 році / Міністерство інфраструктури України. Державна адміністрація залізничного транспорту. Головне управління безпеки руху. - Київ - 2014.

2. Бедняк М.Н., Тахтамышев Х.М. Прогнозирование расхода запасных частей к автомобилям. Киев. «Автомобильный транспорт», 1973, № 10, с. 20-22. 
3. Бокс Дж. Анализ временных рядов. Прогноз и управление.Т1 [Текст]/ Бокс Дж., Дженкинс Г. - М.: Мир, 1974. - 406 с.

4. Галушко В.Г. Вероятностно-статистические методы на автотранспорте. К.:1. Вища школа, 1976. 230 с.

5. Грешилов А. А. Математические методы построения прогнозов / А.А. Грешилов, В. А. Стакун, А. А. Стакун. - М.: Радио и связь, 1997. - 112 с.

6. Єріна А. М. Статистичне моделювання та прогнозування: Навч. посібник / А. М. Єріна - К.: КНЕУ, 2001. - 170 с.

7. Мартинов I. Е. Технічний стан буксових роликопідшипників вантажних вагонів / I. Е. Мартинов // Харківська державна академія залізничного транспорту. Зб. наук. праць. Харків, 2000. - Вип. 41. - С. 38-42.

8. Мартынов И. Э. Анализ опыта эксплуатации цилиндрических роликоподшипников букс грузовых вагонов / И. Э. Мартынов // Вісник Східноукраїнського державного університету. - Луганськ, 2000. - №5 (27). - С. 157-159.

9. Миронов А. А. Буксовый узел тележки - преемственность технологий моделирования при решении задач жизненного цикла / А. А. Миронов, В. П. Ефимов, А. Э Павлюков // Тяжелое машиностроение. - 2005. - № 8. - С. 29-33.

10. Петухов В. М. Прогнозирование остаточного ресурса буксы / В. М. Петухов // 3б. наук. праць. - Донецьк: ДонІ3Т, 2010. - Вип . № 21 - С. 173-177.

11. John Goodman. Technical Assistance Research Program. Affair Study on Complaint Handing in America, 1986. 120 p.

12. Lash M J. The complete Guide to Customer Service. John Wiley and sons, INC. New York, 1996. - 75 p.

Рецензент д-р техн. наук, професор I.Е. Мартинов

Петухов Вадим Михайлович, канд. техн. наук, доиент кафедри вагонів Украӥнської державної академії залізничного транспорту. Тел.: (057) 730-10-35. E-mail: hiitwagon@mail.ru

Депутат Аліна Валеріївна, магістр кафедри вагонів Української державної академї залізничного mpaнcnopmy. E-mail: alina-deputat@mail.ru

Petukhov Vadim Mikhailovich, candidate of technical sciences, associate professor department of wagons Ukraine State Academy of Railway Transport. Tel.; (057) 730-10-35. E-mail: hiitwagon@mail.ru

Deputat Alina Valerevna, student department of wagons Ukraine State Academy of Railway Transport. Email: alina-deputat@mail.ru 